\title{
Magnetic Flux Density Distributions and Discharge Characteristics of a Newly Designed Magnetized Inductively Coupled Plasma
}

\author{
Hee-Woon Cheong* \\ Department of Electrical and Computer Engineering, Seoul National University, Seoul 08826, Korea
}

(Received 9 November 2015, Received in final form 17 December 2015, Accepted 17 December 2015)

\begin{abstract}
Spatial distributions of magnetic flux density in a newly designed magnetized inductively coupled plasma (MICP) etcher were investigated. Radial and axial magnetic flux densities as well as the magnetic flux density on the center of the substrate holder were controllable by placing multiple circular coils around the etcher properly. The plasma density non-uniformity in M-ICP (25 Gauss) can be reduced (1.4\%) compared to that in ICP (16.7\%) when the neutral gas pressure was $0.67 \mathrm{~Pa}$ and a right-hand circularly polarized wave (R-wave) can be propagated in to the etcher by making magnetic flux density increases both radially and axially from the center of the substrate holder.
\end{abstract}

Keywords : M-ICP, plasma density, plasma density non-uniformity, R-wave

\section{Introduction}

The increase in density of semiconductor device integration and the design rule shrinkage make the requirements of dry etchers more stringent. In this regard, a magnetized inductively coupled plasma (M-ICP) etcher [1] which generates high density plasma by applying a weak and controllable magnetic field to the conventional inductively coupled plasma (ICP) etcher [2-5] had been developed. Furthermore, it was confirmed that the performance of M-ICP etcher can be enhanced not only by improving the uniformity of plasma density [6] but also by inducing a right-hand circularly polarized wave (Rwave) [7].

In this paper, two-dimensional magnetic flux density distributions inside the M-ICP etcher were investigated when the magnetic flux density on the substrate holder varies from 4 to 25 Gauss. Plasma density and plasma density non-uniformity characteristics of ICP/M-ICP etcher were also studied according to the magnetic flux density on the center of the substrate holder and the neutral gas pressure. Furthermore, the time-varying magnetic field $\left(B_{z}\right)$ inside the ICP/M-ICP etcher was measured in order to demonstrate that R-wave propagation depends critically

CThe Korean Magnetics Society. All rights reserved.

*Corresponding author: Tel: +82-2-880-9554

Fax:+82-2-872-3005, e-mail: cheong1982@empas.com on the axial magnetic flux density distribution from the center of the substrate holder.

\section{Experimental Setup}

Figure 1 shows the schematic of the ICP/M-ICP etcher. The etcher is powered by two rf generators. One is the source power generator which supplies $27.12 \mathrm{MHz}$ rf

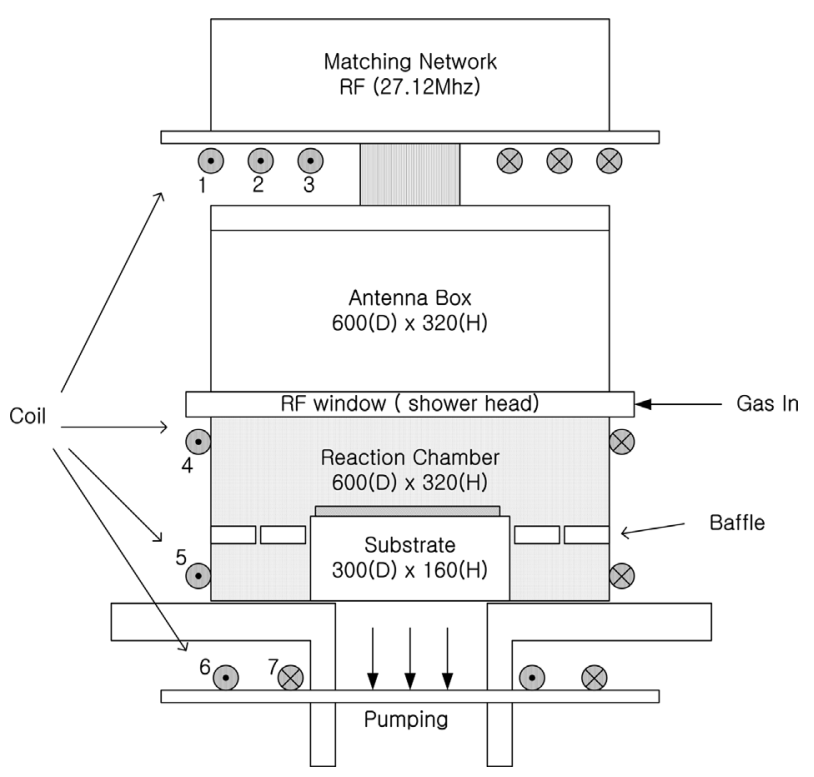

Fig. 1. Schematic of M-ICP etcher with seven circular coils (coil 1, 2, 3, 6, $7: 1400$ turns, coil 4, $5: 1000$ turns). 
power to a two-turn planar antenna and the other is the bias power generator which supplies $2 \mathrm{MHz}$ and/or 13.56 $\mathrm{MHz}$ rf power to the substrate holder whose diameter is $300 \mathrm{~mm}$. A chiller using ethylene glycol as a refrigerant maintains the substrate holder temperature at $15^{\circ} \mathrm{C}$ during the experiments. The etcher is pumped by a $3500 \mathrm{l} / \mathrm{s}$ turbomolecular pump and the base pressure for the experiment was $0.004 \mathrm{~Pa}$. Discharge gas, whose flow rate is regulated by the mass flow controller (MFC), flows into the etcher through a gas inlet and the resultant neutral gas pressure is measured by the hot-filament ionization gauge and baratron gauge (Granville-Phillips ${ }^{\circledR} 392$ MicroIon ${ }^{\circledR}$ Plus). A $20 \mathrm{~mm}$-thick rf window made of aluminabased ceramic is employed to allow the power transfer from antenna to plasma.

Meanwhile, the magnetic fields are generated using seven coils. Firstly, three coaxial coils are placed on top of antenna box. The number of turns of each copper coil is 1400. Secondly, two coaxial coils are located at the bottom of the frame shown in the figure and the number of turns of each coil is also 1400. Lastly, two coils are located at upper and lower sides of the chamber. The number of turns of each coil is 1000 .

The two-dimensional magnetic flux density distributions inside the etcher were simulated with the finite element method magnetic (FEMM) field calculation software when the magnetic flux density on the substrate holder were varied from 4 to 25 Gauss. The experimental measurement of the radial and axial magnetic flux density distributions from the center of the substrate holder were also performed using tesla meter (KANETEC TM-601).

Meanwhile, the radial plasma density distributions inside the ICP/M-ICP etcher were measured using a dual Langmuir probe (DLP) [8]. The vertical height of DLP from the substrate holder was $10 \mathrm{~mm}$. The radial distance of the probe tips from the center of the substrate holder was varied from 0 to $160 \mathrm{~mm}$ at $20 \mathrm{~mm}$ intervals. The plasma density non-uniformity was also calculated using:

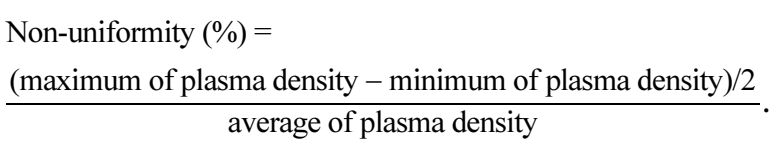

The spatial distributions of the $B_{z}$ of R-wave inside the ICP/M-ICP etcher were also measured using differential magnetic probe (also known as B-dot probe), which is connected directly to a center-tapped transformer to compensate for its alternating current (ac) coupling to electrostatic potential fluctuations [9]. $B_{z}$ is determined by the number of turns of coil (three turns in our experiment) and the coil area (the inner radius of the coil and the coil area are $1.5 \mathrm{~mm}$ and $2.25 \pi \mathrm{mm}^{2}$, respectively, in our

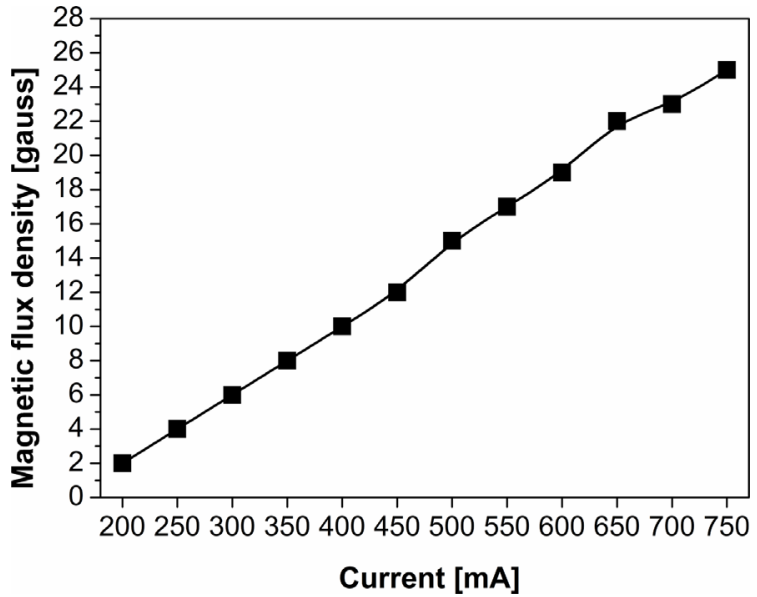

Fig. 2. Magnetic flux density on the center of the substrate holder according to the electric current supplied to coils 1-6 while the fixed electric current was supplied to coil 7.

experiment). The vertical height of B-dot probe from the substrate holder was varied from 10 to $170 \mathrm{~mm}$ at around $10 \mathrm{~mm}$ internals.

\section{Results and Discussion}

Figure 2 shows the magnetic flux density on the center of the substrate holder according to the electric currents supplied to coils 1-6 while the fixed electric current value of $-750 \mathrm{~mA}$ was supplied to coil 7 . The magnetic flux density on the center of the substrate holder where the $\mathrm{Si}$ wafer is placed on is controllable from 0 to 25 Gauss when the electric current of coils 1-6 increases from 0 to $750 \mathrm{~mA}$. Meanwhile, the radial magnetic flux density distributions inside the etcher should be considered carefully because they deeply affect the plasma density non-uniformity [6].

Figure 3 shows two-dimensional magnetic flux density distributions inside the M-ICP etcher. The magnetic flux density vector is perpendicular to the substrate holder and the magnetic flux density increases when the distance from the center of the substrate holder increases radially. It is more clear from Fig. 4, which depicts the radial magnetic flux density distributions according to the electric currents supplied to coils 1-6 while the fixed electric current value of $-750 \mathrm{~mA}$ was supplied to coil 7 , that the magnetic flux density increases radially no matter what the magnetic flux density on the center of the substrate holder is. Based on the fact that the distributions shown in Fig. 4 suppress the flute instability and reduce the plasma density non-uniformity [6], it can be expected that the newly designed M-ICP etcher may exhibit low plasma density non-uniformity regardless of the value of 

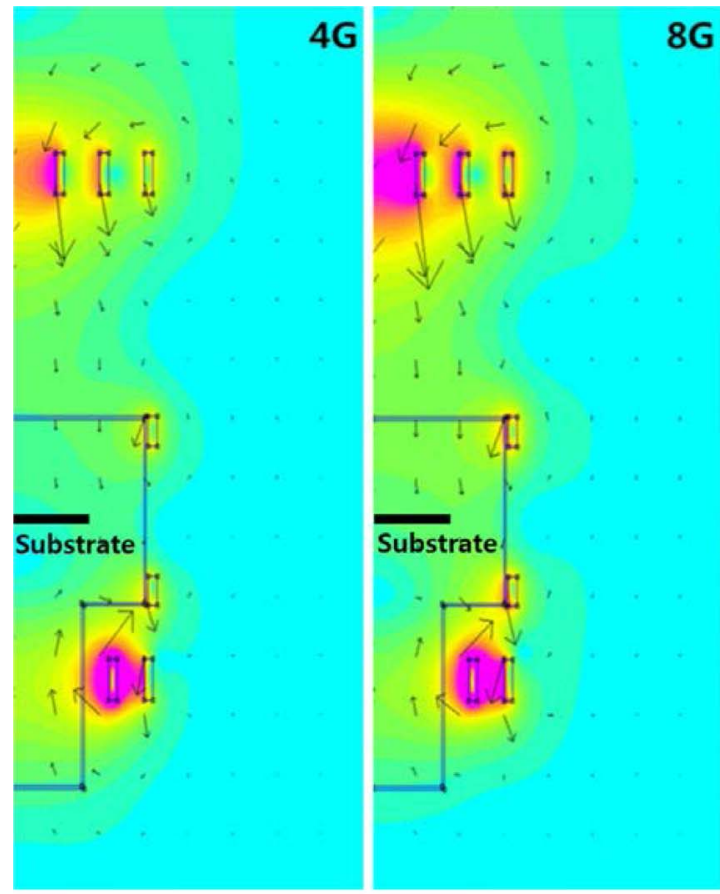

(a)

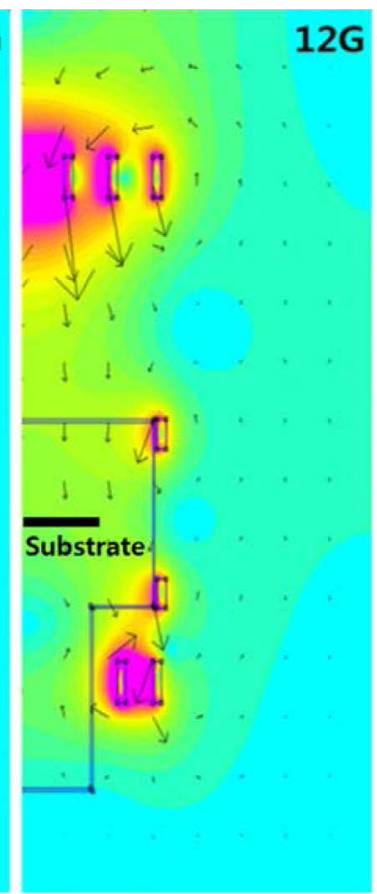

$12 \mathrm{G}$

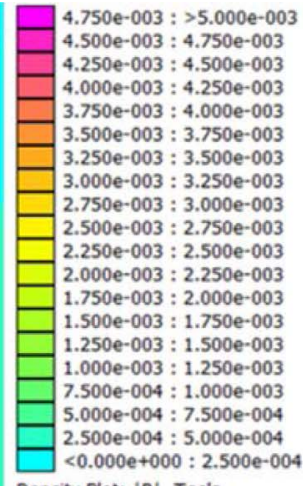

Density Plot: $|8|$, Tesla
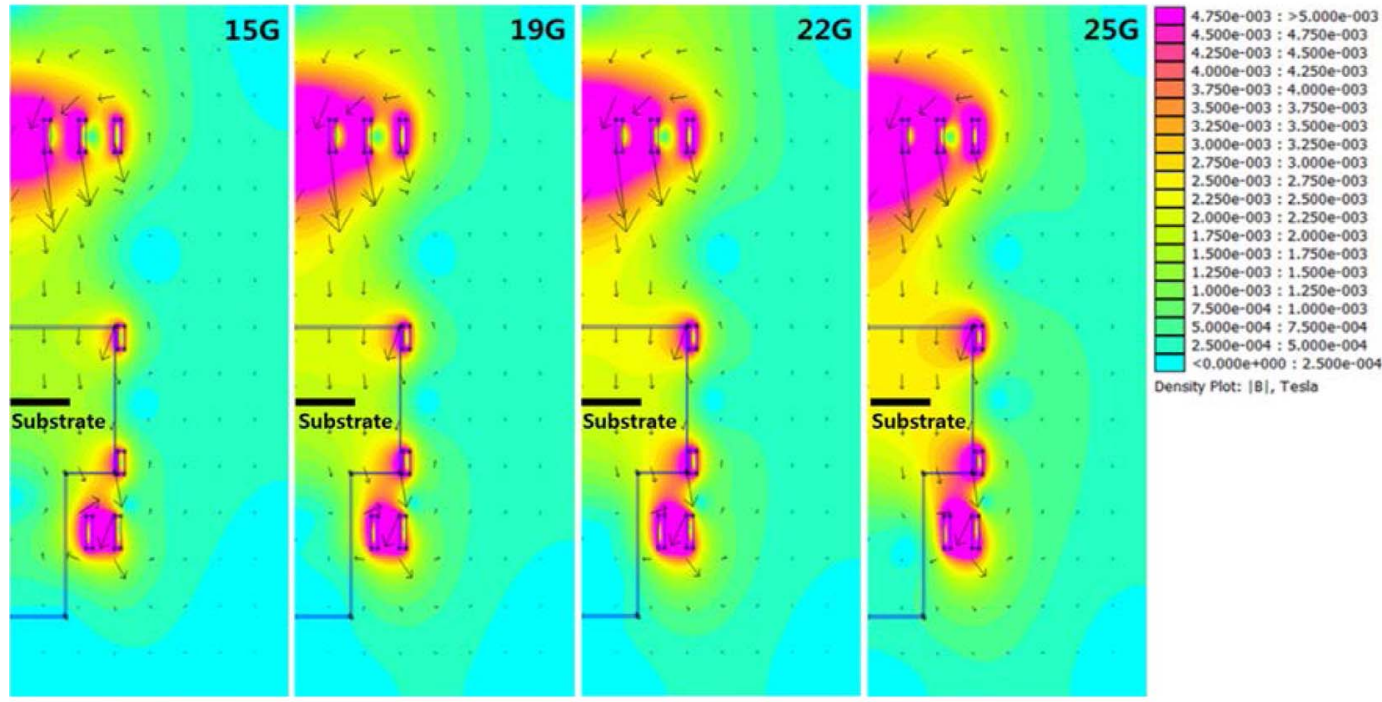

(b)

Fig. 3. (Color online) Two-dimensional magnetic flux density distributions when the magnetic flux density on the center of the substrate is (a) 4 (250 mA to coil 1-6), 8 (350 mA to coil 1-6), 12 Gauss (450 mA to coil 1-6) and (b) 15 (500 mA to coil 1-6), 19 (600 mA to coil 1-6), 22 (650 mA to coil 1-6), 25 Gauss (750 mA to coil 1-6).

the magnetic flux density on the center of the substrate.

Figure 5, Figure 6 and Figure 7 show plasma density, radial plasma density distributions and the calculated radial plasma density non-uniformity inside the ICP/MICP etcher, respectively, according to the magnetic flux density on the center of the substrate holder and the neutral gas pressure. In this experiment, the source power was fixed at $1000 \mathrm{~W}$ and Ar flow rate was fixed at 20 sccm, whereas the magnetic flux density at the center of the substrate holder was varied from 0 to 25 Gauss and the neutral gas pressure was varied from 0.13 to $2.67 \mathrm{~Pa}$. The substrate holder was grounded. The plasma density increases when the magnetic flux density on the center of the substrate holder and/or the neutral gas pressure increases. It is notable that the radial plasma density nonuniformity of M-ICP, no matter what the magnetic flux 


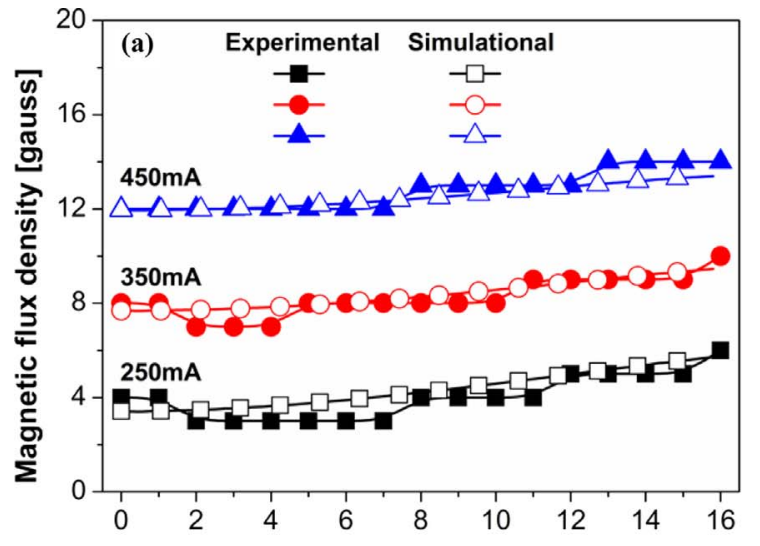

Radial distance from the center of the substrate $[\mathrm{cm}]$

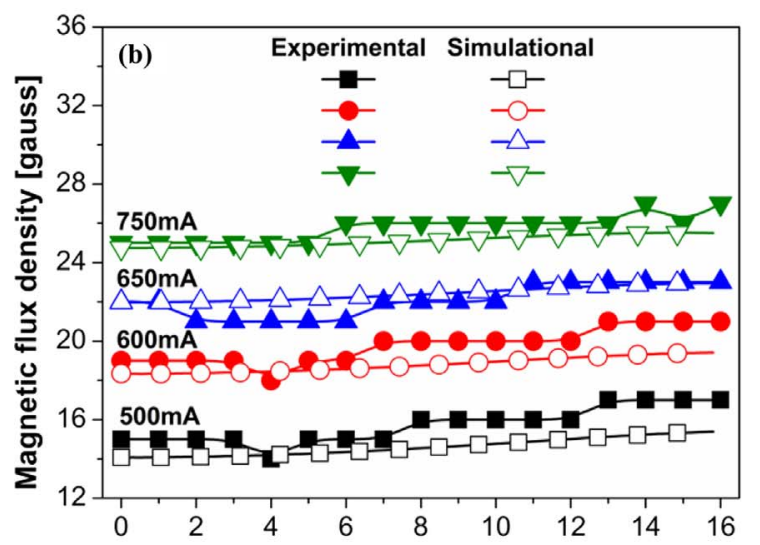

Radial distance from the center of the substrate [cm]

Fig. 4. (Color online) Radial magnetic flux density distributions when the magnetic flux density on the center of the substrate is (a) 4 (250 mA to coil 1-6), 8 (350 mA to coil 1-6), 12 Gauss (450 mA to coil 1-6) and (b) 15 (500 mA to coil 16), 19 (600 mA to coil 1-6), 22 (650 mA to coil 1-6), 25 Gauss (750 mA to coil 1-6).

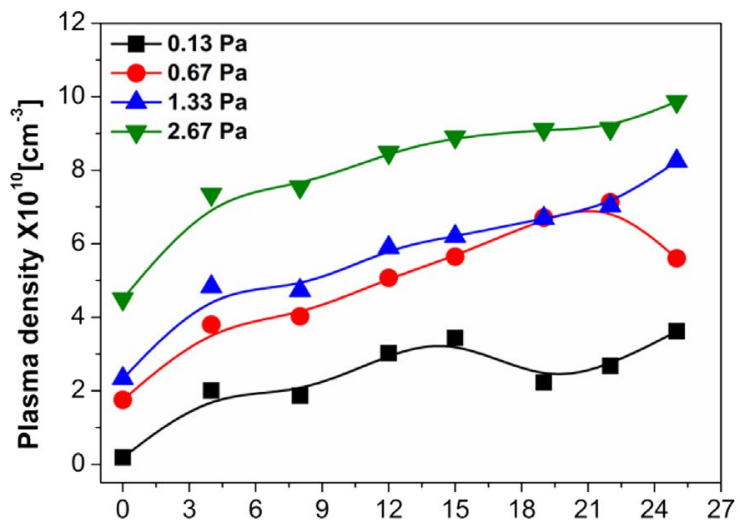

Magnetic flux density on the center of the substrate [gauss]

Fig. 5. (Color online) Plasma density according to the magnetic flux density on the center of the substrate holder and the neutral gas pressure.

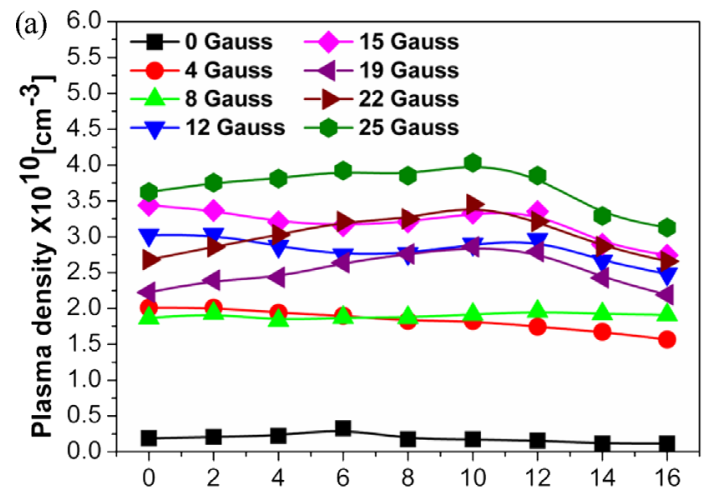

Radial distance from the center of the substrate [cm]
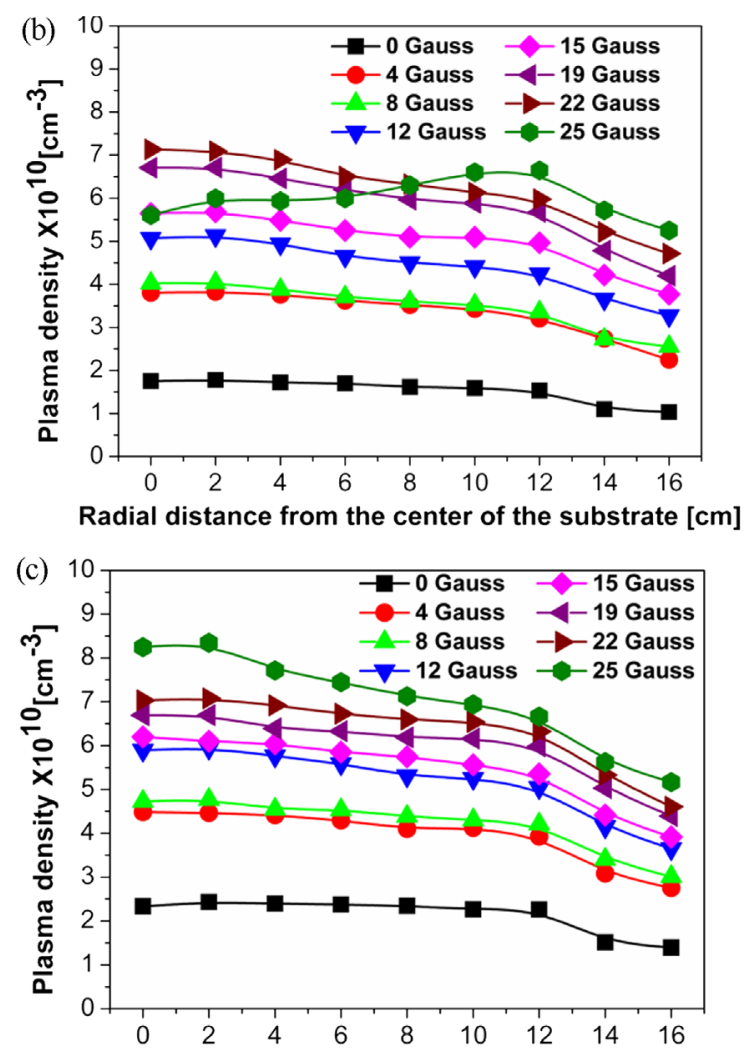

Radial distance from the center of the substrate [cm]

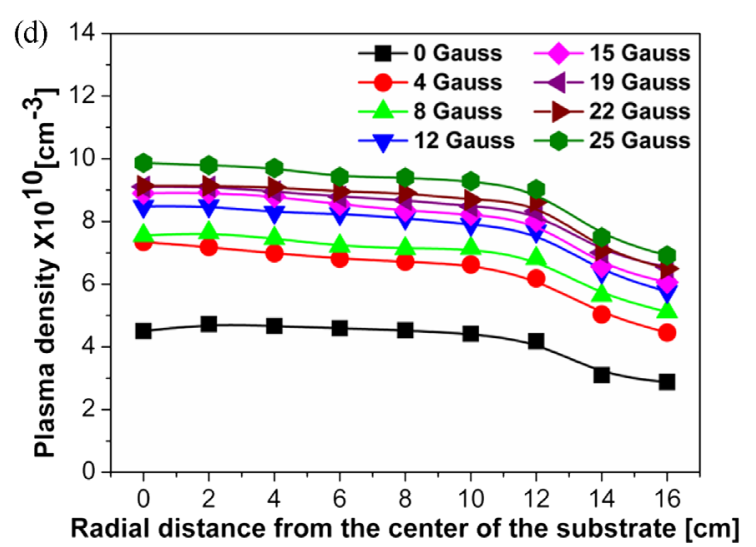

Fig. 6. (Color online) Radial plasma density distributions when the neutral gas pressure is (a) $0.13 \mathrm{~Pa}$ (b) $0.67 \mathrm{~Pa}$ (c) $1.33 \mathrm{~Pa}$ and (d) 2.67 Pa. 


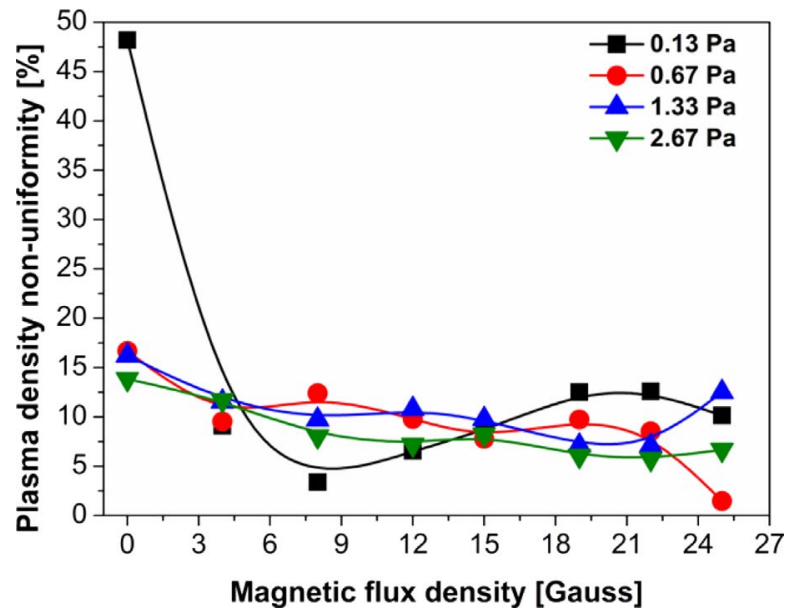

Fig. 7. (Color online) Plasma density non-uniformity according to the magnetic flux density on the center of the substrate holder and the neutral gas pressure.

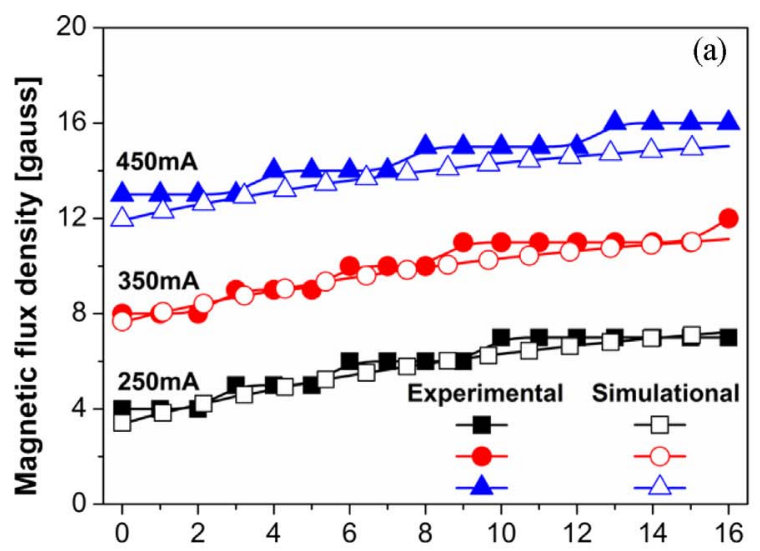

Axial distance from the center of the substrate [cm]

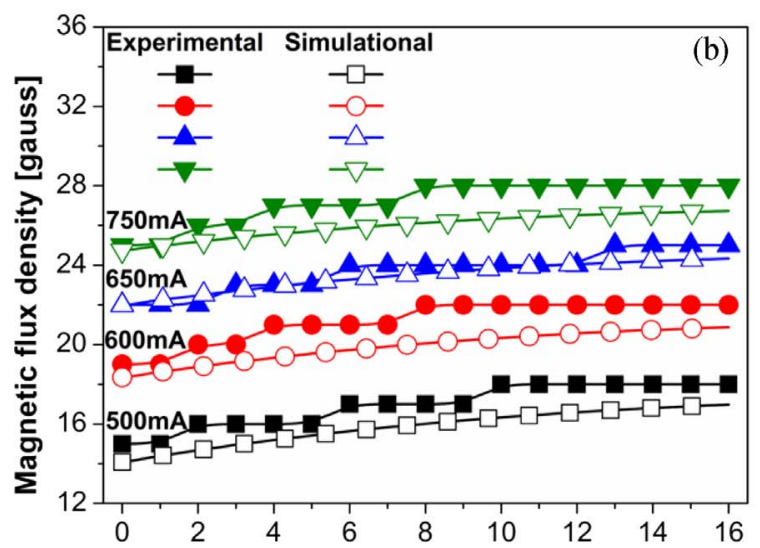

Axial distance from the center of the substrate $[\mathrm{cm}]$

Fig. 8. (Color online) Axial magnetic flux density distributions when the magnetic flux density on the center of the substrate is (a) 4 (250 mA to coil 1-6), 8 (350 $\mathrm{mA}$ to coil 1-6), 12 Gauss (450 mA to coil 1-6) and (b) 15 (500 mA to coil 1-6), 19 (600 mA to coil 1-6), 22 (650 mA to coil 1-6), 25 Gauss (750 mA to coil 1-6). density on the center of the substrate holder is, is lower than that of ICP. More importantly, the lower the neutral gas pressure is, the more remarkable the uniformity improvement effect becomes. Especially, the plasma density non-uniformity was less than $2 \%(1.4 \%)$ when the magnetic flux density on the center of the substrate holder and the neutral gas pressure are 25 Gauss and $0.67 \mathrm{~Pa}$, respectively.

Figure 8 shows the axial magnetic flux density distributions according to the electric currents supplied to coils 1-6 while the fixed electric current value of -750 $\mathrm{mA}$ was supplied to coil 7 . The axial magnetic flux density also increases no matter what the magnetic flux density on the center of the substrate holder is, which is very important for R-wave propagation in M-ICP etcher. $\mathrm{R}$-wave is generated and propagated along the magnetic field if the dispersion relation is satisfied $\left(\omega<\omega_{c e}\right)$ [10]. Figure 9 shows the axial distributions of $B_{z}$ inside the ICP/M-ICP etcher. In this experiment, two types of magnetic flux density distributions were chosen. One is MICP-A, whose magnetic flux density decreases both radially and axially from the center of the substrate holder. The electric current supplied to all coils 1-7 was $200 \mathrm{~mA}$. The other is M-ICP-V, whose magnetic flux density increases both radially and axially from the center of the substrate holder. The electric current supplied to coils 1-6 was $350 \mathrm{~mA}$ while the one supplied to coil 7 was $-750 \mathrm{~mA}$. The magnetic flux density on the substrate for both cases was 8 Gauss. The Ar gas flow was $60 \mathrm{sccm}$ and the neutral gas pressure was $0.67 \mathrm{~Pa}$. The source power and the bias power were $1000 \mathrm{~W}$ and $0 \mathrm{~W}$, respectively. The $B_{z}$ is limited to areas near the rf window and decays rapidly as it penetrates into the plasma within the skin depth in ICP or even in M-ICP-A because the

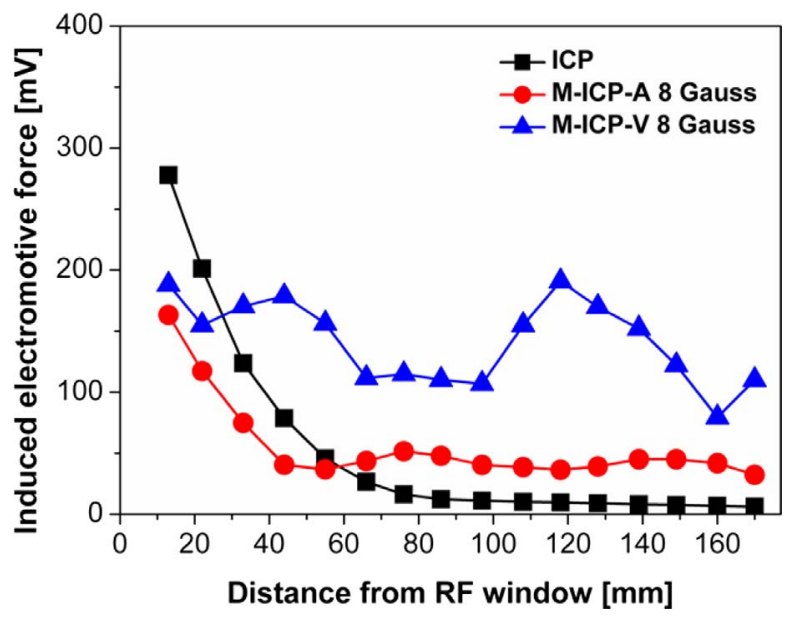

Fig. 9. (Color online) R-wave propagation in ICP, M-ICP-A (8 Gauss) and M-ICP-V (8 Gauss). 
dispersion relation is not satisfied in M-ICP-A, whereas the one is not limited within a skin depth and the energy can be transferred deeper into the plasma in M-ICP-V because the dispersion relation is satisfied and R-wave can be propagated into the etcher in M-ICP-V case.

\section{Conclusions}

Magnetic flux density distributions inside the newly designed M-ICP etcher were investigated. It was confirmed that the magnetic flux density increased radially from the center of the substrate holder, no matter what the magnetic flux density on the center of the substrate holder was. As a result, the flute instability could be effectively suppressed and plasma density non-uniformity in M-ICP (25 Gauss) was significantly reduced (1.4\%) compared to that in ICP $(16.7 \%)$ when the neutral gas pressure was $0.67 \mathrm{~Pa}$. The magnetic flux density also increased axially from the center of the substrate holder no matter what the magnetic flux density on the center of the substrate holder was under the developed system. In doing so, R-wave can be propagated into the M-ICP etcher, which results in the electron heating and the increase in plasma density. After all, controlling the magnetic flux density on the center of the substrate, radial and axial magnetic flux density distributions simultaneously with the proper placement of multiple coils is great advantage of the newly designed $\mathrm{M}-\mathrm{ICP}$ etcher.

\section{References}

[1] H. J. Lee, J. H. Kim, K. W. Whang, and J. H. Joo, J. Vac. Sci. Technol. A 14, 1007 (1996).

[2] S. Imai, J. Vac. Sci. Technol. B 26, 2008 (2008).

[3] B. Wu, A. Kumar, and S. Pamarthy, J. Appl. Phys. 108, 051101 (2010).

[4] F. Karouta, J. Phys. D: Appl. Phys. 47, 233501 (2014).

[5] A. C. Westerheim, A. H. Labun, J. H. Dubash, J. C. Arnold, and H. H. Sawin, J. Vac. Sci. Technol. A 13, 853 (1995).

[6] W. H. Lee, H. W. Cheong, J. W. Kim, and K. W. Whang, Plasma Sources Sci. Technol. 24, 065012 (2015).

[7] H. J. Lee, I. D. Yang, and K. W. Whang, Plasma Sources Sci. Technol. 5, 383 (1996).

[8] Y. Sung, H. B. Lim, and R. S. Houk, J. Anal. At. Spectrom 17, 565 (2002).

[9] C. M. Franck, O. Grulke, and T. Klinger, Rev. Sci. Instrum. 73, 3768 (2002).

[10] M. A. Lieberman and A. J. Lichtenberg, Principles of Plasma Discharges and Materials Processing, John Wiley \& Sons, New Jersey (2005). 